\title{
ÍNDICE DE AGRESIVIDAd ESPACIAL (IAE) DE Chenopodium album EN el Cultivo de Algodón para Santiago del Estero, Argentina ${ }^{1}$
}

\author{
The Space Aggressiveness Index (SAI) for the Chenopodium album in Cotton Crop in Santiago \\ Del Estero, Argentina
}

OCHOA, M.C. ${ }^{2}$, CHAILA, S. ${ }^{2}$ y SOBRERO, M.T. ${ }^{2}$

\begin{abstract}
RESUMEN - E1 Índice de Agresividad Espacial (IAE) es un parámetro bioecológico que indica las características de la distribución de malezas. No existen antecedentes sobre el tema en el cultivo de algodón. El objetivo de este trabajo fue determinar el IAE para Chenopodium album (CHEAL) y relacionarlo con las pérdidas de rendimiento. Se trabajó durante la campaña 2007-2008 en el Campo Experimental de la EEA INTA Santiago del Estero, con el cultivar Guazuncho-INTA. Para el cálculo del IAE, se utilizó un modelo que emplea: altura de planta sobresaliente, su biomasa seca y superficie infestada; número de descendientes, biomasa seca y altura media de los mismos. Dentro del cultivo, en $200 \mathrm{~m}^{2}$, fueron marcadas 10 áreas de $1 \mathrm{~m}^{2}$. Se realizaron análisis estadísticos paramétricos, ANOVA y Tukey $(0,05)$. Los resultados fueron: a) Algodón: peso de parcela medio: 105.000 g; peso por planta: 597 g; el total de plantas: 79,30; altura media: 0,5951 m; rendimiento medio: $2592 \mathrm{~kg} \mathrm{ha}^{-1} \mathrm{y}$, b) CHEAL: peso por parcela: 4920 g; no de plantas por parcela media: 18,7; altura media: 2,72 m; altura de planta madre de maleza: 3,45 m; 187 plantas hijas, medias con 2,40 m de altura y 58 g de biomasa seca, en la parcela de la planta sobresaliente. E1 rendimiento de algodón fue de $2.329,50 \mathrm{~kg} \mathrm{ha}^{-1}$ en el testigo y las pérdidas medias fueron de 89,76\%. E1 IAE obtenido fue de 3,76 (bajo-intermedio) e indica que, en la distribución, actúan numerosas plantas que se desarrollan en torno a una maleza principal. Con un menor IAE, son mayores las pérdidas producidas en el cultivo de algodón.
\end{abstract}

Palabras-clave: CHEAL, distribución, malezas, modelo, rendimiento.

\begin{abstract}
The Space Aggressiveness Index (SAI) is a bio-ecological parameter that depicts weed distribution characteristics. There are not antecedents about this subject in cotton crop. This paper was aimed at determining the space aggressiveness index for Chenopodium album (CHEAL) and relating it to yield losses. Work was done on the Guazuncho-INTA cultivar throughout the 2007-2008 campaign in La Maria Experimental Field, EEA INTA Santiago del Estero. To compute the space aggressiveness index a model that includes the outstanding height, dry biomass, and infected area of the individual plant together with the quantity, dry biomass and average height of the offspring was used. The $200 \mathrm{sq}$ m cropland was divided into ten plots and parametric statistical analyses, ANOVA, and Tukey test $(0,05)$ were made. As to the the results obtained were: a) Cotton: -average weight per plot: 105.000 g; -weight per plant: 597 g; total number of plants: 79, 30; average height:

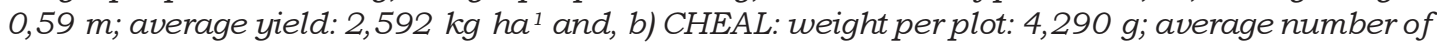
individuals per plot: 18,7; average height: 2,72 m; parent plant height: 3,45 $\mathrm{m}$ whereas for the offspring the figures were: 187 individuals; average height: 2, $40 \mathrm{~m}$; average dry matter: $58 \mathrm{~g}$ in plot of surpassing plant. Cotton yield was 2,329,50 kg ha-1 and crop losses were of 89, 76\%. The space aggressiveness index obtained was that of 3,76 (i.e. low-intermediate). Such an index indicates that numerous plants growing around a main weed act together in distribution. The lower space aggressiveness index, the higher yield losses occur in cotton crop.
\end{abstract}

Keywords: CHEAL, distribution, model, weed, yield.

1 Recebido para publicação em 6.11.2010 e aprovado em 31.3.2011.

2 Cátedra de Matología, Facultad de Agronomía y Agroindustrias-UNSE, Av. Belgrano (S) 1912, Santiago del Estero, Argentina, $<$ mcochoa@unse.edu.ar> 


\section{INTRODUCCIÓN}

La infestación de malezas es uno de los principales factores bióticos presentes en un agroecosistema de algodón e interfiere en el desarrollo y productividad del cultivo, tanto en sistemas de siembra convencional como en siembra directa (Salgado et al., 2002; Freitas et al., 2006).

$\mathrm{El}$ algodón, presenta un crecimiento inicial lento (Freitas et al., 2006), metabolismo $\mathrm{C}_{3}$ y un sistema radical que se ubica superficialmente durante los primeros 40 dias desde su emergencia (DDE), es afectado por la competencia de malezas (Beltrão, 1996; Salgado et al., 2002). Por otra parte, las altas temperaturas y precipitaciones durante su desarrollo, favorecen la presencia de malezas (Brach, 2005; Ledda, 2005). La convivencia del cultivo de algodón con las malezas, durante todo el ciclo de cultivo, puede provocar reducciones de la producción, que varian desde un $68 \%$ hasta un $95 \%$, y producir inconvenientes en la cosecha (Salgado et al., 2002). Pueden, además, ocasionar pérdidas de más del 90\% en el rendimiento de fibra, por lo cual es necesario mantener el cultivo libre de malezas durante el período crítico de competencia (PCC). En siembras convencionales, el PCC se encuentra entre los 8 a 66 días desde la emergencia (DDE) (Beltrão, 2004; Freitas et al., 2006). En áreas de riego, como la zona estudiada, este período puede extenderse hasta los 80 DDE (Guimaraes et al., 2007). En siembras de surcos estrechos, este período comprende 30 DDE (Guevara, 2005).

Entre las malezas de hoja ancha más importantes en el cultivo se encuentran Ipomoea nil, Portulaca oleracea, Sida spp., Malvastrum coromandelianum, Chenopodium album, Amaranthus quitensis, Senna occidentalis, Trianthema portulacastrum (Guevara, 2005; Ledda, 2005; Sobrero et al., 2005). Chenopodium, Amaranthus, Portulaca y Malvastrum aparecen en la primera etapa del ciclo de cultivo, desde mediados de octubre hasta mediados de diciembre, en Santiago del Estero (Sobrero et al., 2005). Chenopodium album (CHEAL) es una maleza cosmopolita, anual, de dias cortos, colonizadora exitosa de nuevas áreas, que exhibe una gran plasticidad en respuesta al ambiente (Bhowmik \& Reddy, 1988). Se adapta a un rango de temperaturas de 6.5 a $44.5{ }^{\circ} \mathrm{C}$ (Huang et al., 2001). Esta especie modifica su respuesta fotosintética a cambios en la intensidad de la luz (Ballaré et al., 1987; Colquhoun et al., 2001). La plasticidad en la tasa de crecimiento de tallos le permite minimizar la interferencia de otras especies (Moechnig et al., 2003a). El efecto de la longitud del día también hace que las malezas de semillas pequeñas, como las de CHEAL, compitan con los cultivos de semillas más grandes, debido a que sus plántulas tienen mayores tasas de crecimiento relativo al principio que la estación de crecimiento de los cultivos en los que crecen (Harbur \& Owen, 2004). E1 conocimiento de las malezas, sus características biológicas y su respuesta al ambiente, están consideradas como herramientas básicas para la correcta elección del programa de manejo de malezas (Brach, 2005). Asimismo, la determinación de un indice permite caracterizar las estrategias competitivas de crecimiento de las malezas (Moechnig et al., 2003b).

E1 Índice de Agresividad Espacial (IAE) es un parámetro bioecológico que permite establecer diferencias entre especies de malezas en cuanto a su dominio sobre un territorio invadido, ya sea en áreas naturales o cultivadas, desde que llegan al área, y los progresos en la colonización o competencia por recursos (Chaila et al., 2006). No hay, en el cultivo de algodón, un índice que determina agresividad de las malezas que compiten con él, con relación a su capacidad de distribución en el espacio. El objetivo del presente trabajo fue determinar el IAE para CHEAL y comparar su comportamiento poblacional en el cultivo de algodón con relación a las pérdidas en el rendimiento.

\section{MATERIAL Y MÉTODOS}

El ensayo se desarrolló en el campo de la Estación Experimental Agropecuaria - INTA

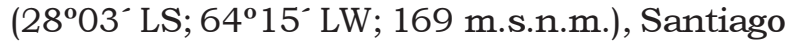
del Estero, Argentina, entre noviembre de 2007 y mayo de 2008. La siembra del lote se efectúo el 15 de noviembre de 2007, con el cultivar de algodón Guazuncho-INTA. La distancia entre surcos fue de 0,50 m y se determinó el número de plantas en 10 por metro lineal. Luego de la 
emergencia del cultivo, se determinaron dos

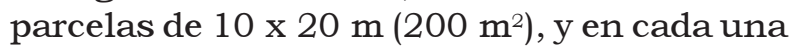
de ellas, se marcaron 10 parcelas de $1 \mathrm{~m}^{2}$, que se mantuvieron libres de malezas durante todo el ciclo (TL), y parcelas en las que se dejó crecer plantas de CHEAL, y donde se eliminó, de forma manual, el resto de las malezas, tarea que se repitió hasta el cierre del canopeo. En estas parcelas, durante el mes de noviembre de 2007, se marcaron las plantas sobresalientes de CHEAL. Luego de las labores básicas de raleo, el área demarcada, salvo el riego y la aplicación de insecticidas, no se efectuaron otras labores. La cosecha se llevó a cabo el 24 de abril de 2008.

Para la extracción de muestras de CHEAL, se establecieron, al azar, 10 parcelas de $1 \mathrm{~m}^{2}$. En cada una de ellas, se evalúo: a) En el cultivo de algodón: peso medio de parcela $\left(\mathrm{g} \mathrm{m}^{-2}\right)$ y por planta (g por planta), número total de plantas, altura media de plantas $(\mathrm{m})$, rendimiento medio $\left(\mathrm{kg} \mathrm{ha}^{-1}\right)$. b) Sobre CHEAL: peso por parcela $\left(\mathrm{g} \mathrm{m}^{-2}\right)$, promedios de número de plantas por parcela, altura de plantas (m), altura de planta sobresaliente $(\mathrm{m})$, número de plantas hijas, altura media de plantas hijas (m) y peso seco medio $(\mathrm{g})$.

Para el cálculo del IAE de CHEAL, se empleó un modelo simple, cuya expresión, que interrelaciona la altura de la planta sobresaliente (Hpm), su biomasa seca (BSpm), la superficie infestada relacionada con el número de plantas hijas o descendientes que invaden el área en estudio alrededor de la planta sobresaliente $\left(A_{t}\right)$, la biomasa seca media y la altura media, se indica seguidamente:

$$
I A E=\frac{(H p m \times B S p m) A_{t}}{(n+1)\left[\sum \frac{b s}{n_{t}} \sum \frac{h_{t}}{n_{t}}\right]\left(T_{1}-T_{2}\right)} \times 100
$$

donde $H p m$ = altura de la planta sobresaliente; $B S p m$ = biomasa seca de la planta sobresaliente; $(n+1)=$ número de descendientes $+1 ; A t=$ superficie de estudio; $n t=$ número total de descendientes; $b s=$ biomasa seca media de las plantas del área; $h t=$ altura media de todas las plantas del área; $T_{1}=$ tiempo de aparición de la planta original; $T_{2}=$ tiempo de aparición de la última planta medida.
Se compararon los distintos grados de infestación mediante ANOVA, incluso del IAE $\mathrm{y}$, cuando se hizo necesario, se realizó un test de Tukey $(\mathrm{p}<0,05)$.

Los datos meteorológicos fueron obtenidos de la Estación Agrometeorológica del Instituto Nacional de Tecnología Agropecuaria (INTA), Santiago del Estero, Argentina.

\section{RESULTADOS Y DISCUSIÓN}

En el momento de la cosecha, las plantas de CHEAL, en las diez parcelas, superaban la altura de las de cultivo. La altura media en nueve de las diez parcelas evaluadas fue mayor a 2 m. Según Holm et al. (1977), CHEAL puede alcanzar los $3 \mathrm{~m}$ de altura en cultivos como el maíz y el sorgo en condiciones de suelo fértil y buen contenido hídrico.

Cuando el algodón creció en surcos estrechos con Amaranthus quitensis (AMAQU) (datos no mostrados), su altura media fue de 2,73 m. La capacidad de CHEAL, cuando crece con otras especies, de continuar incrementando su altura, incluso después de haber alcanzado el IAF máximo, puede atribuirse a la combinación de competencia intra e interespecífica por luz. Según Colquhoun et al. (2001), estos investigadores, al estudiar en cámaras de crecimiento el efecto de la temperatura y del fotoperíodo sobre la longitud de tallos, encontraron que la altura de los mismos se incrementaba con el aumento de la temperatura, siendo $1,55 \mathrm{~m}$ y $0,26 \mathrm{~m}$, con fotoperiodos de 16 y 8 horas de luz, respectivamente. En las condiciones de este ensayo, se registraron temperaturas similares a las mencionadas por Huang et al. (2001) y Roman et al. (1999), que le permitieron alcanzar y superar en altura al cultivo, aunque la emergencia de maleza y cultivo hayan sido simultáneas (Tabla 1).

En condiciones de campo y sin cultivo, Shrestha \& Swanton (2007), determinaron alturas máximas de $1,30 \mathrm{~m}$ y $0,83 \mathrm{~m}$, en plantas que emergieron desde mediados hasta fines de la primavera, asociando la elongación de tallos a la acumulación de temperaturas, y señalaron que la fecha de plantación afectó la altura final de CHEAL. Aumentos de altura, producidos por elevadas tasas de crecimiento en estadios tempranos de desarrollo, en 
Tabla 1 - Temperaturas registradas durante el ciclo de crecimiento del cultivo y Temperaturas base, óptima y máxima de elongación de tallos de CHEAL $^{\underline{1}}$. EEA INTA La María, Santiago del Estero, Argentina, 2007-2008

\begin{tabular}{|c|c|c|c|c|c|c|c|c|}
\hline $\mathrm{T}^{\mathrm{o}} \mathrm{Mes}$ & Nov. 2007 & Dic. 2007 & Ene. 2008 & Feb. 2008 & Mar. 2008 & Abr. 2008 & \multicolumn{2}{|c|}{$\begin{array}{c}\mathrm{T}^{\mathrm{o}} \text { elongación de tallos de } \\
\text { CHEAL }\end{array}$} \\
\hline Tmax & 32,4 & 33,4 & 33,0 & 32,5 & 29,4 & 26,6 & $\mathrm{~Tb}$ & 6,5 \\
\hline Tmin & 15,2 & 18,4 & 20,2 & 18,9 & 17,8 & 12,3 & Topt & 20,5 \\
\hline Tmed & 24,3 & 25,8 & 26,3 & 25,2 & 23,3 & 19,3 & Tmax & 37,0 \\
\hline
\end{tabular}

1/ Temperaturas máximas (Tmax), mínimas (Tmin) y medias (Tmed) en ${ }^{\circ} \mathrm{C}$ en comparación a las temperaturas base (Tb), óptima (Topt) y máxima (Tmax), para la elongación del tallo de CHEAL (Huang et al., 2001; Roman et al., 1999).

respuesta a la temperatura, y ante la presencia de plantas vecinas de su misma o diferente especie, son consideradas estrategias competitivas (Radosevich et al., 1997). La plasticidad en la tasa de crecimiento de tallos permiten minimizar la interferencia de otras especies (Moechnig et al., 2003b).

Estas características ambientales que actúan sobre la maleza permiten una mayor altura de sus tallos, contribuyendo al incremento de la biomasa total en la superficie ocupada. Al lograr mayor altura de la planta madre, se afecta uno de los componentes importantes del modelo que es la altura de la planta sobresaliente $(\mathrm{Hpm})$ y la altura total de plantas del área $\left(h_{t}\right)$. Grandes valores de Hpm y de biomasa seca total (Bspm) con la superficie que ocupan las especies At tendrán influencia sobre la expresión total del IAE. Esto justifica valores finales o promedios altos del indice. Además, de los efectos sobre la biomasa total, la altura determina una mejor y mayor superficie de distribución de las semillas provenientes de la planta madre y aumenta la densidad y, por lo tanto, los efectos de la infestación del área y la competencia con el cultivo.

E1 número de plantas de CHEAL por parcela varió entre 7 y 28, densidades que representan pérdidas significativas para el cultivo. En un experimento similar, de algodón en surcos estrechos creciendo con CHEAL y AMAQU, bajo las mismas condiciones de este ensayo, las densidades de CHEAL variaron de 3 a 15 plantas $\mathrm{m}^{-2}$. Sin embargo, en otros cultivos y ambientes, las densidades de CHEAL fueron mucho mayores que las de este trabajo. Así, Cardina et al. (1996), en campos sembrados con soja, bajo dos sistemas de cultivo, sin labranza y con labranza convencional, observaron que la emergencia de plántulas fueron de 7 a 400 y de 2 a 476 plántulas $\mathrm{m}^{-2}$, según las condiciones ambientales del año considerado. En cebada, en diferentes años se registraron las máximas pérdidas de rendimiento en el cultivo, un $23 \%$ con densidades de 150 plantas $\mathrm{m}^{-2}$ y de $36 \%$ con 300 a 400 plantas $\mathrm{m}^{-2}$ (Conn \& Thomas, 1987). La competitividad de CHEAL estaría determinada por la densidad, el tiempo relativo de emergencia de la maleza y del cultivo y las condiciones ambientales (Fisher et al., 2004).

Para el cálculo del Índice, la densidad actuaría, afectando estructuralmente el proceso de la determinación, con variantes importantes conforme el número en $(n+1)$ y en la sumatoria general con $n_{t}$ que es el número total de descendientes. Estos aspectos actúan sustancialmente en la agresividad de la maleza y por ende en la competencia sobre el cultivo y permiten subir o bajar los valores de IAE. Es importante notar que las variaciones ocurren de acuerdo a los cambios considerados, como superficie total (At) que, muchas veces, es fija $y$, otras, variables según se determine la parcela previamente o que se la mida en un área de reciente infestación con la maleza.

La biomasa seca de las plantas sobresalientes (BSpm) y la biomasa seca media de las plantas del área (bs) son resultantes directos de la agresividad de las especies sobre el cultivo, expresan los niveles de competencia e intervienen en el modelo, afectando los componentes altura y densidad que intervienen directamente sobre el indice, produciendo cambios importantes en sus valores.

En la Tabla 2, se expresan los promedios de pesos por parcela y por planta, el número de plantas y la altura media del cultivo y de la maleza en la cosecha. 
Tabla 2 - Pesos promedios por parcela y por planta, número de plantas y altura media de algodón y CHEAL en la cosecha

\begin{tabular}{|l|c|c|c|c|}
\hline & $\begin{array}{c}\text { Peso promedio } \\
\text { de parcela (g) }\end{array}$ & $\begin{array}{c}\text { Peso } \\
\text { promedio } \\
\text { por planta } \\
(\mathrm{g})\end{array}$ & $\begin{array}{c}\mathrm{N}^{\mathrm{o}} \mathrm{de} \\
\text { plantas }\end{array}$ & $\begin{array}{c}\text { Altura } \\
\text { media } \\
(\mathrm{m})\end{array}$ \\
\hline Algodón & 105.000 & 597,00 & 79,30 & 0,59 \\
\hline CHEAL & 4.920 & 66,10 & 18,70 & 2,72 \\
\hline
\end{tabular}

En este ensayo, el peso seco promedio de CHEAL, para densidades de 7 y más de 20 plantas $\mathrm{m}^{-2}$, fueron de 0,55 y de $2,15 \mathrm{~g} \mathrm{~m}^{-2}$. Estos resultados coinciden con los hallados por Bhowmik \& Reddy (1988), quienes observaron que el peso fresco de CHEAL aumentó cuando se incrementó la densidad, desde $26.360 \mathrm{~kg}$ ha ${ }^{1}$ con 16 plantas $\mathrm{m}^{-1}$ hasta $46.000 \mathrm{~kg} \mathrm{ha}^{-1}$ con 64 plantas $\mathrm{m}^{-1}$. Este tipo de respuesta podría deberse a competencia intraespecífica. Por lo tanto, incrementos en la densidad producen plantas más pequeñas, menor número de inflorescencias, menor producción de semillas y reducción del peso seco (Holm et al., 1977). Huang et al. (2001), al estudiar, en plantas que crecian aisladas, el efecto del fotoperiodo sobre la biomasa por planta, determinaron pesos secos medios de $10 \mathrm{~g}$ y $1,2 \mathrm{~g}$, con 16 y 8 horas de luz, respectivamente, a los 33 DDE. La biomasa final de plantas estaria asociada, también, a la fecha de emergencia, ya que fue mayor en plantas que emergieron a mediados de primavera, como ocurrió en este trabajo (Shrestha \& Swanton, 2007). El rendimiento medio del algodón fue de $2.592 \mathrm{~kg} \mathrm{ha}^{-1}$, un $50 \%$ inferior al rendimiento comercial, $4.291,7 \mathrm{~kg} \mathrm{ha}^{-1}$, obtenido en ensayos de surcos estrechos $\left(0,50 \mathrm{~m}\right.$ entre líneas y 10 plantas $\left.\mathrm{m}^{-1}\right)$ sin competencia de malezas en el área de riego (Mondino \& Peterlin, 2005).

La Tabla 3 muestra los valores obtenidos para CHEAL de biomasa seca media por planta y por parcela, la altura de la planta sobresaliente entre las 187 plantas totales, y también se expresa la altura media de las plantas y su desvío entre las 20 plantas de la parcela $\mathrm{n}^{\circ} 9$ del ensayo, en donde se encontraba la planta sobresaliente.

El modelo utilizado permitió calcular el IAE y las pérdidas alcanzadas por competencia específica en el rendimiento del algodón y los valores de los indices encontrados para las diferentes densidades de la maleza, que se detallan en la Tabla 4. E1 Índice de Agresividad Espacial obtenido para altas densidades del ensayo fue 3,76 (bajo-intermedio).

Los indices obtenidos son intermedios. Pueden señalar desde un daño grave al cultivo (89,76\% de pérdida) hasta un daño significativo (37,67\% de pérdida) medidos en la cosecha. Si bien, no presentan diferencias entre si para las áreas o parcelas de infestación alta, media y baja, producen pérdidas de rendimiento que difieren estadísticamente. Los sistemas de siembra de algodón estrechos y ultraestrechos pretenden disminuir la incidencia de las malezas, debido a que cubren el suelo durante 30 días, comparados a los 60-75 días de los sistemas convencionales. En Santiago del Estero, durante los primeros 30 y 80 días desde la siembra (DDS), se produce el flujo de emergencia de CHEAL, independientemente de la distancia entre líneas de cultivo (Sobrero et al., 2005). Esta etapa coincide con el período crítico de competencia (PCC) establecido para el cultivo.

Tabla 3 - Parámetros indicativos de la planta sobresaliente de CHEAL

\begin{tabular}{|l|c|}
\hline $\begin{array}{l}\text { Biomasa seca media por planta de la } \\
\text { parcela de la planta sobresaliente }\left(\mathrm{n}^{\mathrm{o}} \text { 9) }\right.\end{array}$ & $58 \mathrm{~g}$ \\
\hline $\begin{array}{l}\text { Biomasa seca media de la parcela de la } \\
\text { planta sobresaliente }\left(\mathrm{n}^{\mathrm{o}} \text { 9) }\right.\end{array}$ & $1.150 \mathrm{~g}$ \\
\hline Altura de planta sobresaliente (parcela $\mathrm{n}^{\circ}$ 9) & $3.45 \mathrm{~m}$ \\
\hline $\mathrm{N}^{\mathrm{o}}$ de plantas hijas del total del ensayo & 187 \\
\hline $\begin{array}{l}\text { Altura media de plantas en la parcela de la } \\
\text { planta sobresaliente }\left(\mathrm{n}^{\mathrm{o}} \text { 9) }\right.\end{array}$ & $2,40 \mathrm{~m} \pm 0,91$ \\
\hline
\end{tabular}

Tabla 4 - Índice de Agresividad Espacial de CHEAL en el cultivo de algodón, Santiago del Estero, RA, 2007-2008

\begin{tabular}{|c|c|c|c|c|}
\hline \multirow[b]{2}{*}{$\begin{array}{l}\text { Grado de } \\
\text { infestación }\end{array}$} & \multicolumn{2}{|c|}{ CHEAL } & \multicolumn{2}{|c|}{ Algodón } \\
\hline & $\begin{array}{c}\text { Densidad } \\
\left(\text { plantas } \mathrm{m}^{-2}\right)\end{array}$ & IAE & Pérdida\% &  \\
\hline Muy alta & $\geq 100$ & $3,76609 \mathrm{a}$ & 89,76 & $265,43 \mathrm{a}$ \\
\hline Alta & 28 & $3,20491 \mathrm{~b}$ & 76,39 & $612,00 \mathrm{~b}$ \\
\hline Media & 16 & $3,19637 \mathrm{~b}$ & 56,15 & $1.137,00 \mathrm{c}$ \\
\hline Baja & 7 & $3,17104 \mathrm{~b}$ & 37,70 & $1.616,00 \mathrm{~d}$ \\
\hline
\end{tabular}

* Letras iguales no presentan diferencias para Tukey $(\mathrm{p}<0.05)$. 
Es conocida la capacidad del algodón de compensar rendimientos al modificar la separación entre hileras. Por ello, estos rendimientos pueden no afectarse (Brach, 2005). Sin embargo, malezas como CHEAL, debido a que los mayores flujos de emergencia se producen en los primeros 30 días desde la siembra del cultivo, a su rápido crecimiento inicial y a las temperaturas favorables para la elongación de tallos, superan la altura del cultivo y afectan su rendimiento, incluso en sistemas de surcos estrechos (Sobrero et al., 2005). El IAE está en función de la distribución espacial y presentará variaciones al aumentar la superficie infestada, la altura de la planta principal y su biomasa. En esta especie las semillas, caen alrededor de la planta madre y originan un crecimiento en manchones en los cultivos (Holm et al., 1977). Con una mayor altura de la planta sobresaliente, es mayor la dispersión de semillas en el espacio ocupado $\mathrm{y}$ en áreas vecinas. Al aumentar el número de las plantas vecinas en el área, la altura y la biomasa medias de las plantas disminuirá el IAE. Un índice bajo indica que en la distribución actúan numerosas plantas, que se desarrollan en torno a una planta principal o sobresaliente. E1 desarrollo de estrategias alternativas, basadas en el conocimiento de la biología y ecología de CHEAL, es necesario, particularmente, en bajas densidades de malezas, lo que es común en sistemas de cultivos (Colquhoun et al., 2001). Los resultados de este ensayo coinciden con 1o expresado por Gramid \& Stoltenberg (2009) respecto a que esta maleza incrementa su altura, la biomasa de tallos y disminuye la biomasa de hojas cuando crece bajo el canopeo de un cultivo. Además, CHEAL exhibe gran plasticidad morfológica, que siempre le permite producir semillas que aseguran su persistencia en sistemas de cultivo (Anderson, 2008). Una mejor comprensión de esta plasticidad podría ayudar a explicar los conceptos de período crítico para la remoción de malezas y de umbral económico para su manejo.

\section{AGRADECIMIENTOS}

Este Trabajo se llevó a cabo gracias al Subsidio de Secyt-Unse

\section{LITERATURA CITADA}

ANDERSON, R. L. Weed seedling and survival as affected by crop canopy. Weed Technol., v. 22, n. 4, p. 736-740, 2008.

BALLARÉ, C. L. et al. Early detection of naighbour plants by phytochrome perception of spectral changes in reflected sunlight. Plant Cell Environ., v. 10, n. 7, p. 551-557, 1987

BELTRÃO, N. E. M. Cultivos de plantas oleaginosas y de fibras algodón. In: LABRADA, R; CASELEY, J. C.; PARKER, C. (Eds.) Manejo de malezas para países en desarrollo. Roma: FAO, 1996. p. 359-362.

BHOWMIK, P. C.; REDDY, K. N. Interference of common lambsquarters (Chenopodium album) in transplanted tomato (Lycopersicon esculentum). Weed Technol., v. 2, n. 4, p. 505-508, 1988.

BRACH, A. Monitoreo de malezas en el cultivo de algodón en el Norte Santafesino. In: Proyecto Nacional Algodón.

Informe de avance $\mathbf{N}^{\circ} \mathbf{1 .} \mathbf{2}^{\mathbf{0}}$ Reunión Anual. Agosto 2005.

Sáenz Peña, Chaco, Argentina: INTA EEA, 2005. p. 93-95.

CARDINA, J.; SPARROW, D. H.; McCOY, E. L. Spatial relationships between seedbank and seedling populations of common lambsquarters (Chenopodium album) and annual grasses. Weed Sci., v. 44, n. 2, p. 298-308, 1996.

COLQUHOUN, J.; STOLTENBERG, D.E.; BINNING, L.K.; BOERBOOM, C.M. et al. Phenology of common lambsquarters growth parameters. Weed Sci., v. 49, n. 2, p. 177-183, 2001.

CONN, J. S.; THOMAS, D. L. Common lambsquarters (Chenopodium album) interference in spring barley. Weed Technol., v. 1, n. 4, p. 312-313, 1987.

CHAILA, S. et al. Spatial agresiveness index (Iae) o Flaveria videntis (L.) o Kuntze for sugar cane crops of three Tucuman, Argentine. Localities Biocell, v. 30, p. 177, 2006.

FISHER, D. W.; GORDON HARVEY, R.; BAUMAN, T.T.; S.E.HART, G.A.; JOHNSON, J.J. KELLS, P.; WESTRA; LINDQUIST, J. Common lambsquarters (Chenopodium album) interference with corn across the northcentral United States. Weed Sci., v. 52, n. 6, p. 1034-1038, 2004.

FREITAS, R. S.; BERGER, P.G.; FERREIRA, L.R.; SILVA, A.C.; CECON, P.R.; SILVA, M.P. Manejo de plantas daninhas na cultura do algodoneiro em sistema da Plantio Direto. Planta Daninha, v. 24, n. 2, p. 339-346, 2006.

GUEVARA, G. Encuesta sobre problemática de malezas en el cultivo de algodón en Chaco-Formosa. In: Proyecto Nacional Algodón. Informe de Avance $N^{\circ}$ 1. $2^{\circ}$ Reunión Anual. Agosto 2005. Sáenz Peña, Chaco, Argentina: INTA EEA, 2005. p. 101-104.

GRAMID G. G.; STOLTENBERG, D. E. Adaptative responses of field-grown common lambsquarters (Chenopodium album) to variable light quality and quantity environments. Weed Sci., v. 57, n. 3, p. 271-280, 2009. 
GUIMARAES, S. C.; HRYCYK, M. F.; MENDONÇA, E. A F. Efeito de fatores ambientais a seletividade do alachlor ao algodoneiro. Planta Danihna, v. 25, n. 4, p. 813-821, 2007.

HARBUR, M. M.; OWEN, M. D. K. Light and growth rate effects on crop and weed responses to nitrogen. Weed Sci., v. 52, n. 4 , p. $578-583,2004$.

HOLM, L. G.; PLUCKNETT, D.L.; PANCHO, J.V. Y HERBERGER, J.P. Chenopodium album L. Chenopodiaceae, goosefoot family. In: The World's Worst Weeds: distribution and ecology. Honolulu:University Press of Hawaii, 1977. p. 84-91.

HUANG, J. Z.; SHRESTHA, A.; TOLLENAAR, M.; DEEN, W.; RAHIMIAN, H; SWANTON, C.J. Effect of temperatura and photoperiod on the phenological development of common lambsquarters. Weed Sci., v. 49, n. 4, p. 500-508, 2001.

LEDDA, A. Control químico de malezas en el cultivo de algodón. In: Proyecto Nacional Algodón. Informe de Avance No ${ }^{1.2}{ }^{\circ}$ Reunión Anual. Agosto 2005. Sáenz Peña, Chaco, Argentina: INTA EEA, 2005. p. 124-126.

MOECHNIG, M. J.; BOERBOOM, C. M.; STOLTENBERG, D. E.; BINNING, L. K. Growth interactions in communities of common lambsquarters (Chenopodium album) and giant foxtail (Setaria faberi), and corn in mixed communities. Weed Sci., v. 51, n. 3, p. $363-370,2003 a$.

MOECHNIG, M. J.; STOLTENBERG, D. E.; BOERBOOM, C. M.; BINNING, L. K. Empirical corn yield loss estimation from common lambsquarters (Chenopodium album) and giant foxtail (Setaria faberi) in mixed communities.. Weed Sci., v. 51, n. 3, p. 386-393, 2003 b.
MONDINO, M.; PETERLIN, O. Efectos de la densidad de plantas sobre la productividad de algodones sembrados en surcos distanciados a 0,50 m. In: Proyecto Nacional Algodón. Informe de Avance $N^{\circ}{ }^{1 .} 2^{\circ}$ Reunión Anual. Agosto 2005. Sáenz Peña, Chaco, Argentina INTA EEA, 2005. p. 34-37.

RADOSEVICH, E. S.; HOLT, J. S; GHERSA, C. M. Responses of crops and weeds to competition. In: RADOSEVICH, E. S.; HOLT, J. S; GHERSA, C. M. Weed ecology, implications for management. 2.ed. New York: John Wiley \& Sons, 1997. p. 194-199.

ROMAN, E. S.; GORDON THOMAS, A.; MURPHY, S.D.; SWANTON, C.J. Modeling germination and elongation of common lambsquarters (Chenopodium album). Weed Sci., v. 47 , n. 3, p. 298-308, 1999

SALGADO, T. P.; ALVES, P.L.C.A.; MATTOS, E.D.; MARTINS, J.F.; D.D, HERNANDEZ. Periodos de interferencia das plantas dañinas na cultura do algodoneiro (Gossypium hirsutum). Planta Daninha, v. 20, n. 3, p. 373379, 2002.

SHRESTHA, A.; SWANTON, C. J. Parameterization of the phenological development of select annual weeds under noncropped field conditions. Weed Sci., v. 55, n. 5, p. 446454, 2007.

SOBRERO, M. T.; CHAILA, S.; OCHOA, M.C.; EPSTEIN, M.F.; TARGA,M.G. Relevamiento de malezas. In: Proyecto Nacional Algodón. Informe de Avance $N^{0} 1.2^{\circ}$ Reunión Anual. Agosto 2005. Sáenz Peña, Chaco, Argentina: INTA EEA, 2005. p. 105-107. 